\title{
Synthesis of Perfluoroalkylated Bulky Triarylamines
}

\author{
Bassam Alameddine, ${ }^{a}$ Corinne Savary, ${ }^{b}$ Olivier Aebischer, ${ }^{b}$ Titus A. Jenny*b \\ a Chemistry Department, University of Balamand, P.O. Box 100, Tripoli, Lebanon \\ b Chemistry Department, University of Fribourg, 9 chemin du Musée, 1700 Fribourg, Switzerland \\ Fax +41(26)3009739; E-mail: titus.jenny@unifr.ch
}

\begin{abstract}
The synthesis of two new triarylamine compounds bearing perfluoroalkylated side chains is described. Good thermal stabilities combined with a blue emission make these compounds promising candidates for materials applications.
\end{abstract}

Key words: triarylamines, perfluoroalkylation, cross-coupling, blue emission, amorphous materials

Triarylamines have found wide applicationin devices that require properties like the generation, the transport, and the recombination of cationic or anionic charges such as organic light emitting diodes (OLED), ${ }^{1,2}$ photorefractive systems (PR), ${ }^{3}$ and solar cells, ${ }^{4}$ besides their use in electrophotographic devices like photocopiers and laser printe $r \sim$ These widespread applications are due to the low oxidation potential of the nitrogen atom which, upon hole injection, generates a stable triarylamine radical cation necessary for the hoping mechanism. ${ }^{6}$ The difficulty, however, resides in the deposition of a monocrystalline thin film to avoid the formation of defects and grain boundaries that act as traps for the charge carriers. As an alternative approach, the deposition of an amorphous thin film is used. Moreover, the major limitation of arylarnines in these applications is their short lifetime because of the significant degradation caused by impurities and fatigue due to morphological changes during the device operation. Therefore, a high thermal stability, and the ability to form an amorphous phase are the two main properties necessary for the employment of a triarylamine product for such devices?

$N, N^{\prime}$-Diphenyl- $N, N^{\prime}$-( $m$-tolyl)benzidine (TPD) has been first reported ${ }^{\mathrm{s}}$ as an efficient hole transporting material for electroluminescent(EL) devices, due to its ability to form an amorphous phase. Since then, various papers have been published on the synthesis of new nitrogen-containing aryl products ranging from low molecular weight molecules? to starbust assemblies, ${ }^{10,11}$ oligomers, ${ }^{12}$ dendrimers $^{13}$ as well as polymers. ${ }^{14}$ We wish to report herein a new strategy to achieve amorphous phases, simply by the addition of peripheral perfluorinated chains to relatively small $\mathrm{N}$-containing aryl products. The perfluorinated chains are generally known to weaken the van der Waals interactions leading to reduction of the lateral intermolecular aggregation and, consequently, to a macroscopic amorphism. ${ }^{15}$ Additionally, the inert behavior of the perfluorinated chains in terms of chemical reactivity bestows the molecule with a better thermal stability.

The perfluoroalkylated starting materials 7 and $\mathbf{8}$ were synthesized by applying the most convenient Heck crosscoupling reaction conditions: ${ }^{16}$ the naphthylamine derivatives 1 and 2 were first transformed into their respective arenediazoniurn salts ${ }^{1}{ }^{1}$ in quantitative yield. These stable and isolable tetrafluoroborate salts were reacted in a subsequent step with an appropriate commercially available perfluoroalkene, in the presence of a catalytical amount of palladium(II) acetate under ligandless conditions, affording the fluorinated alkene compounds 5 and 6 in 79 and $90 \%$ yields, respectively, after short reaction times. The hydrogenation of the brominated naphthalene derivative 5 was achieved by $\mathrm{Rh} / \mathrm{C}$ catalyst, under 50 bar pressure of hydrogen, affording the perfluoroalkylatednaphthalene 7 in $91 \%$ yield. On the other hand, the nitronaphthylated alkene 6 was hydrogenated in presence of a $\mathrm{Pd} / \mathrm{C}$ catalyst reducing both the nitro and the olefin groups, yielding the desired product 8 in $94 \%$ yield (Scheme 1).

The reaction between the naphthylamine moiety 8 with two-fold excess of the brominated naphthalene 7 employing the palladium-catalyzedamination reaction under the

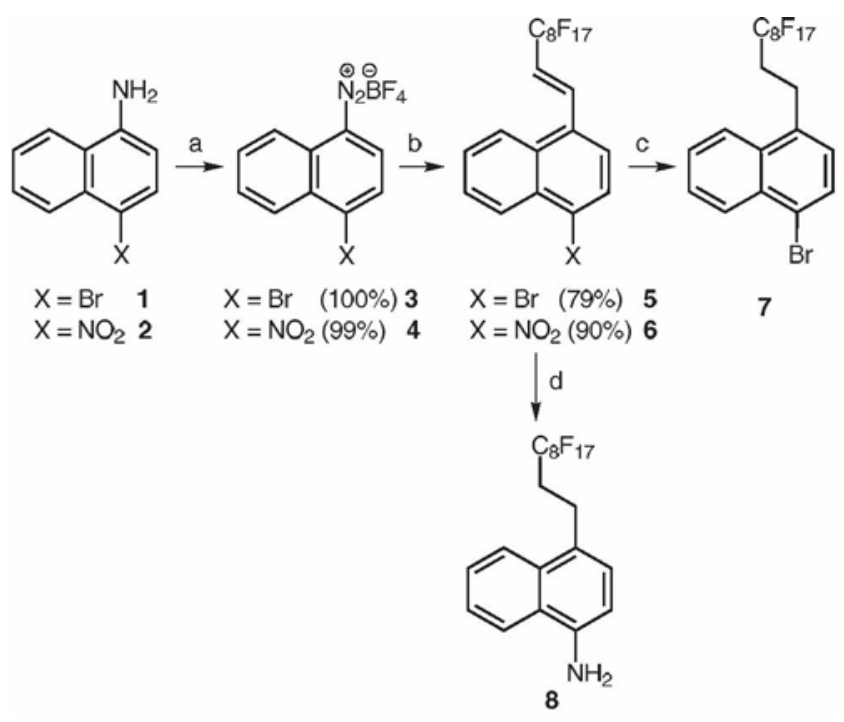

Scheme 1 Synthesis of the perfluoroalkylatednaphthalene building blocks 7 and 8. Reagents and conditions: a) $\mathrm{BF}_{3} \cdot \mathrm{OEt}_{2}, t-\mathrm{BuNO}_{2}$, DME; b) $1 H, 1 H, 2 H$-perfluorodecene, $\left.\mathrm{Pd}(\mathrm{OAc})_{2}, \mathrm{MeOH}, 40^{\circ} \mathrm{C} \mathrm{c}\right)$ $\mathrm{Rh} / \mathrm{C}, 50$ bar $\mathrm{H}_{2}, \mathrm{CH}_{2} \mathrm{Cl}_{2}$, r.t., 1 d, $91 \%$; d) $\mathrm{Pd} / \mathrm{C}, 3$ bar $\mathrm{H}_{2}, \mathrm{MeOH}-$ THF, r.t., $1 \mathrm{~d}, 94 \%$. 
<smiles>[2H]c1ccc(Br)c2ccccc12</smiles><smiles>[R]c1ccc(N)c2ccccc12</smiles>

$\mathrm{R}=\left(\mathrm{CH}_{2}\right)_{2} \mathrm{C}_{8} \mathrm{~F}_{17}$<smiles>[R]c1cccc2c(N(c3ccc([R])c4ccccc34)c3ccc([R])c4ccccc34)cccc12</smiles>

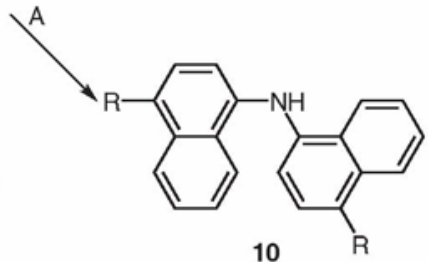

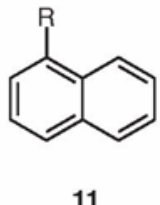

11

Scheme 2 Attempted synthesis of the perfluoroalkylated trinaphthylamine 9 via the one pot Pd-catalyzed amination reaction. Reagents and conditions: $\mathrm{Pd}(\mathrm{OAc})_{2}, t-\mathrm{B} \mathrm{u}_{3} \mathrm{P}, t$-BuONa, toluene, reflux.

mild Buchwald-Hartwig conditions ${ }^{18}$ (Scheme 2) only yields the perfluoroalkylated binaphthylarnine 10. Increasing the reaction time resulted in the formation of trace amount of the debrominated species $\mathbf{1 1}$ along with the binaphthylamine derivative 10. No trace of the perfluoroalkylated trinaphthylamine derivative 9 was found despite corresponding claims in the patent literature. . $^{19,20}$

Thus, the cross-coupling reaction between an equimolar mixture of $\mathbf{7}$ and $\mathbf{8}$ yields the building block $\mathbf{1 0}$ in $\mathbf{7 1 \%}$ yield. The palladium-catalyzed amination reaction between $\mathbf{1 0}$ and the perfluoroalkylated bromonaphthalene 7 using several bases like, sodium tert-butoxide, cesium carbonate, and sodium hydroxide does not lead to the formation of any product at room temperature, while it affords a mixture of the debrominated naphthalene $\mathbf{1 1}$ and the naphthalene-naphthalene homocoupling product $\mathbf{1 2}$ when the reaction mixture was heated at reflux (Scheme 3). When performing the more reactive lithiated derivative of 10 by exchange of the acidic proton by reaction with $n-\mathrm{BuLi}$, the subsequent reaction with 7 under suitable conditions ${ }^{13,21,22}$ yields exclusively the debrominated perfluoroalkylatednaphthalene $\mathbf{1 1}$.
We believe that steric hindrance caused by the two bulky naphthalene groups prevents the third perfluoroalkylated naphthyl species to bind to the nitrogen atom and, hence, leads to attack at the non-activated carbon-5 position. Only few patents describe the synthesis of the parent trinaphthylamine $9 \mathrm{~m}=\mathrm{H})^{19,20}$ characterized by elemental analysis and mass spectrometry, methods which do not allow for distinction between $\mathbf{9}$ and $\mathbf{1 2}$.

Applying the Buchwald-Hartwig conditions, the binaphthylarnine derivative $\mathbf{1 0}$ and either the perfluoroalkylated bromobenzene or 4,4'-dibromobiphenyl, affords the desired triarylamine derivatives $\mathbf{1 3}$ and $\mathbf{1 4}$ in 64 and 57\% yield, respectively (Scheme 4).

Both products are isolated as colorless solids showing relatively good solubility in common organic solvents. The $\mathrm{W}$ - v is spectra of $10^{-3} \mathrm{M}$ solutions of $\mathbf{1 3}$ and $\mathbf{1 4}$ in THF reveal the presence of two absorption bands for each compound: The former at 267 and $349 \mathrm{~nm}$ whereas the latter has the maxima at 301 and $367 \mathrm{~nm}$. Measuring the fluorescence of both compounds at the same concentration $\left(10^{-3} \mathrm{M}\right.$ in THF) reveals their blue emission nature: the excitation of $\mathbf{1 3}$ at $349 \mathrm{~nm}$ yields an emission peak at 430

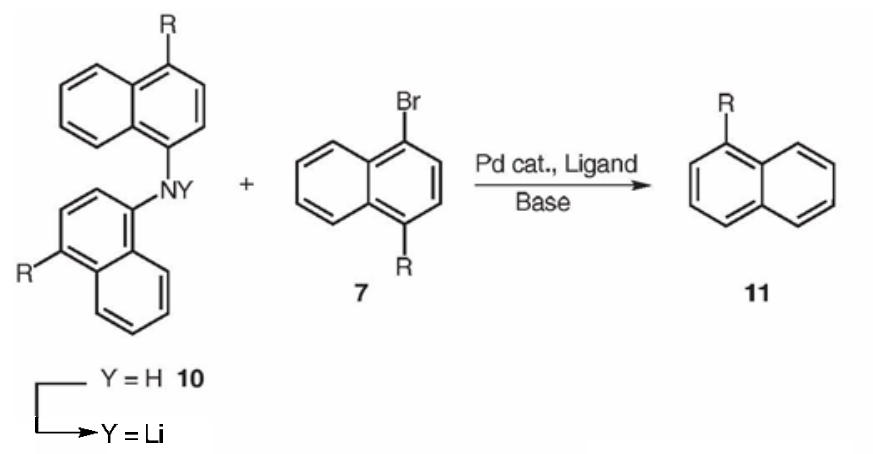<smiles>[R]c1ccc(Nc2ccc([R])c3c([Z])cccc23)c2ccccc12</smiles>

12

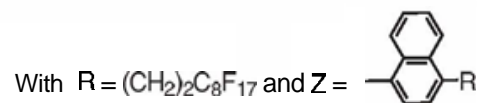

Scheme 3 Attempted synthesis of the perfluoroalkylated trinaphthylamine 9 using the binaphthylamine (or amide) as a starting material. 


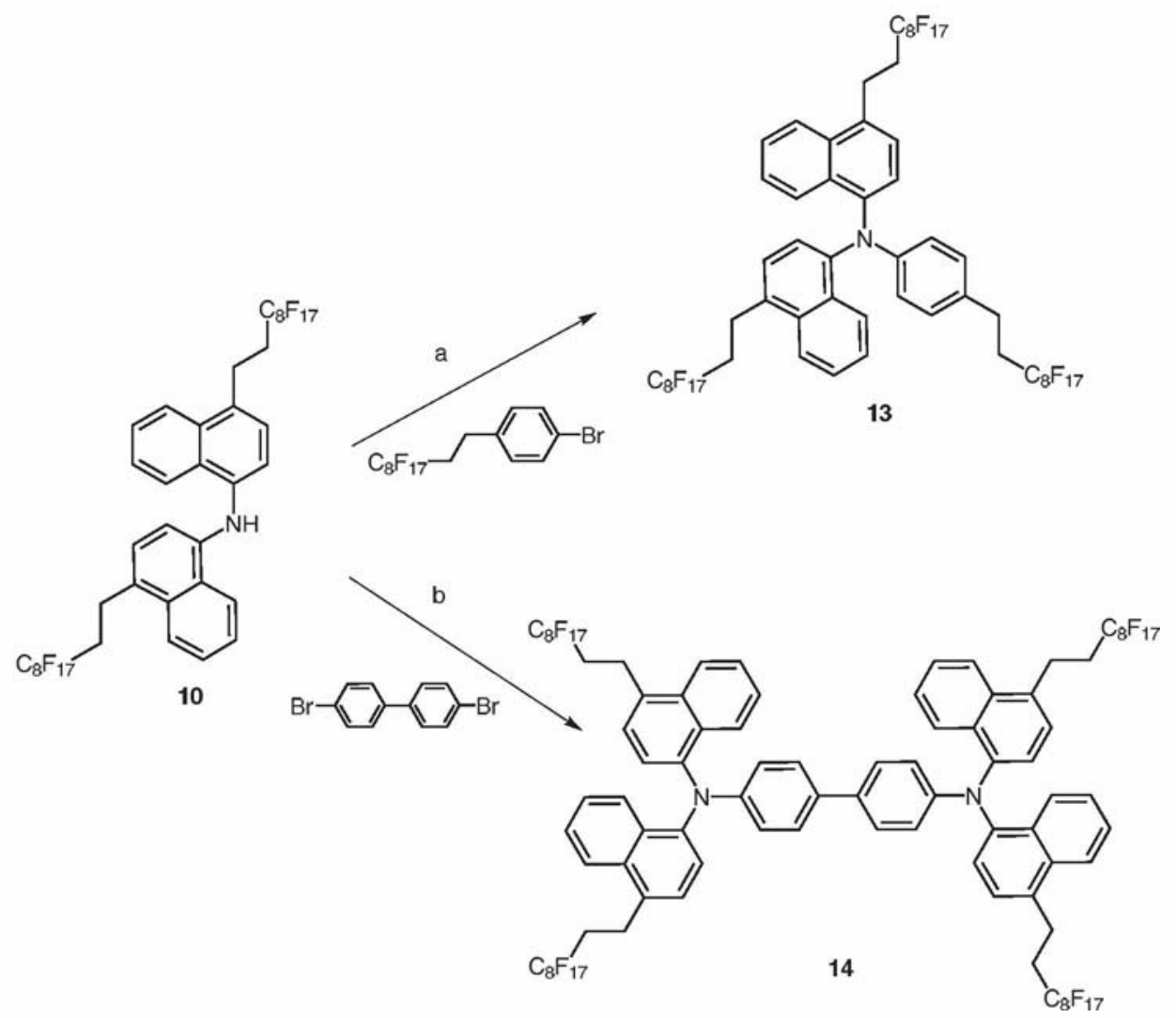

Scheme 4 Synthesis of the perfluoroalkylatedarylamine derivatives 13 and 14. Reagents and conditions: $\mathrm{Pd}(\mathrm{OAc})_{2}, t$ - $\mathrm{Bu} \mathrm{u}_{3} \mathrm{P}, t$-BuONa, toluene, reflux: a) $3.5 \mathrm{~d}, 64 \%$; b) $7 \mathrm{~d}, 57 \%$.

$\mathrm{nm}$, whereas the fluorescence spectrum of $\mathbf{1 4}$ shows an emission peak at $455 \mathrm{~nm}$ upon irradiating the solution at 367 nm (Figure 1).

Interestingly, the perfluoroalkylated triarylamine 13, isolated from synthesis as a transparent, amorphous waxy solid, was found to produce fibers even at room temperature. The investigation of such a fiber drawn at room temperature, under a polarized optical microscope, reveals a long homogeneous structure showing aligned features (Figure 2), a behavior which is normally observed for polymer melts. We therefore conclude that $\mathbf{1 3}$ aggregates

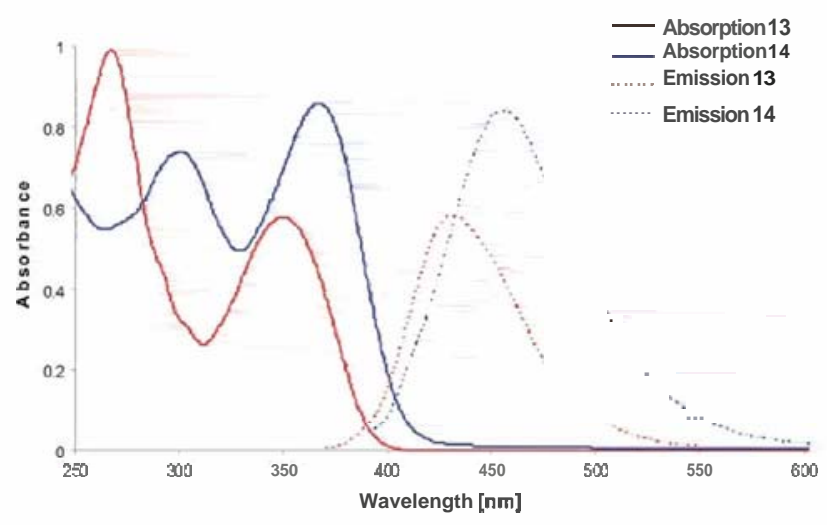

Figure 1 UV-vis spectra of 13 (red) and 14 (blue). Normalized emission spectra of $\mathbf{1 3}$ (dotted red) and $\mathbf{1 4}$ (dotted blue) in THF. in one dimension to a supramolecule, thereby mimicking a much larger species.

The preliminary differential scanning calorimetry (DSC) investigation of the thermal properties of 13 and 14 reveal very puzzling properties: product $\mathbf{1 3}$ remains glassy down to $-20^{\circ} \mathrm{C}$ and does not melt even at high temperature $\left(-450^{\circ} \mathrm{C}\right)$, while the triarylamine derivative, $\mathbf{1 4}$ exhibits a melting point at a relatively high temperature $\left(242^{\circ} \mathrm{C}\right)$.

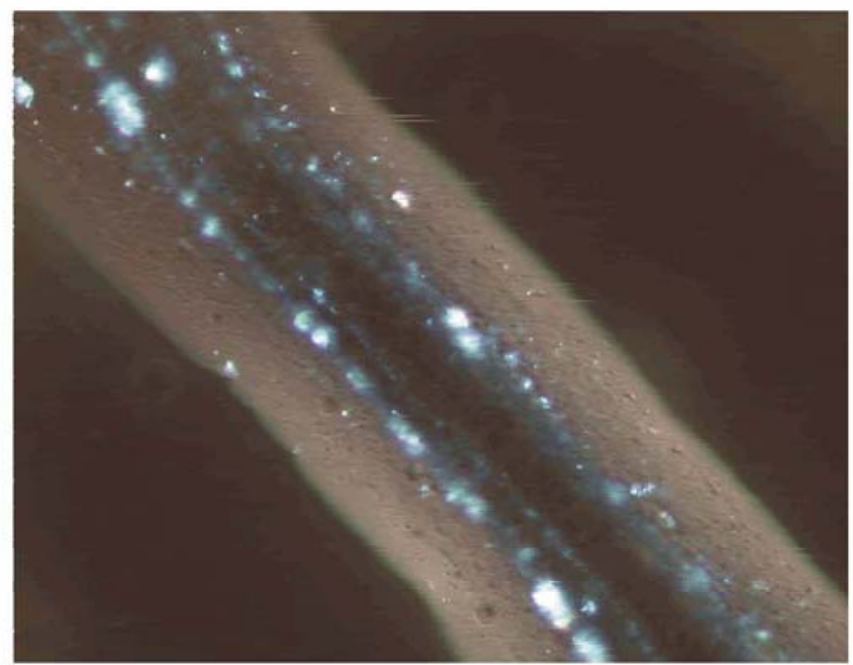

Figure 2 POM micrograph $(x 100)$ of a fiber of $\mathbf{1 3}$ drawn at room temperature. 
Finally, significant thermal stability was also observed since both products were heated several times up to $450^{\circ} \mathrm{C}$ without causing any degradation.

In conclusion, new triarylamine compounds bearing perfluoroalkylated side chains were synthesized in good yields. The amorphous nature of the products was introduced, by design, by the addition of peripheral perfluoroalkylated chains well known for their weak van der Waals interaction. These materials excel by their excellent thermal stability over a wide temperature range allowing them to be used for applications as blue-emissive materials.

NMR spectra were recorded with Bruker Avance DRX 500 and Bruker Avance DPX 360 spectrometers. Chemical shifts are referred to TMS as an internal standard. TLC analyses were done using aluminum sheets coated with silica gel $60 \mathrm{~F}_{254}$. Column chromatography was carried out using Merck silica gel $60(0.04$ $0.063,230-400$ mesh). Electron impact (EI) and electrospray ionization (ESI) mass spectra (MS) were recorded on a Vacuum Generators Micromass VG 70/70E spectrometerand on a FT/ICR mass spectrometer Bruker 4.7T BioApex II. Differential scanning calorimetry traces were recorded using a Mettler Toledo DSC822e, calibrated with indium $\left(\mathrm{mp} 156.6^{\circ} \mathrm{C}, \Delta \mathrm{H}_{\mathrm{f}}=28.45 \mathrm{KJ} \mathrm{mol}^{-1}\right)$ before each series of measurements. Polarization optical microscopy (POM) was carried out using an Axioscope Zeiss microscope; the photographs were taken with a Fujix Digital camera HC-300Z.

I-Bromo-4-(3,3,4,4,5,5,6,6,7,7,8,8,9,9,10,10-heptadecafluorodecyl)benzene was prepared according to literature ${ }^{16}$ starting from 4 bromophenylamine. All the other chemical reagents were purchased from commercial sources. Solvents were dried by passing them under argon through a special purification system similar to the one proposed by Grubbs. ${ }^{23}$ The solvents were saturated with argon for 15-30 min prior to use.

\section{Diazotization; 4-Bromonaphthalene-1-diazoniumTetrafluo- roborate (3); Typical Procedure}

$\mathrm{BF}_{3} \cdot \mathrm{OEt}_{2}(2.5 \mathrm{~mL}, 19.5 \mathrm{mmol})$ was placed in a three-necked roundbottomed flask equipped with an addition funnel, a septum, and a reflux condenser. The temperature was lowered to $-15^{\circ} \mathrm{C}$ and a solution of 4-bromonaphthylamine $(\mathbf{1} ; 3 \mathrm{~g}, 13 \mathrm{mmol})$ in DME $(15 \mathrm{~mL})$ was added dropwise first, followed by the addition of a solution of $t$-BuNO $(2.1 \mathrm{~mL}, 15.6 \mathrm{mmol})$ in DME $(25 \mathrm{~mL})$ at the same temperature during $15 \mathrm{~min}$. After stirring the mixture at $-15^{\circ} \mathrm{C}$ for $20 \mathrm{~min}$, the temperature was raised to $5^{\circ} \mathrm{C}$ over aperiod of $20 \mathrm{~min}$. Pentane $(50 \mathrm{~mL})$ was then added and the suspended compound was collected by suction filtration, washed with $\mathrm{Et}_{2} \mathrm{O}\left(50 \mathrm{~mL}\right.$ at $0{ }^{\circ} \mathrm{C}$, and 50 $\mathrm{mL}$ at r.t.) yielding a green-bmwn solid $(4.16 \mathrm{~g},-100 \%)$.

\section{4-Nitronaphthalene-1-diazoniumTetrafluoroborate(4)}

Prepared following the diazotization reaction described above: 4-nitronaphthylamine $(2 ; 2.52 \mathrm{~g}, 13 \mathrm{mmol}), \mathrm{BF}_{3} \cdot \mathrm{OEt}_{2}(2.5 \mathrm{~mL}, 19.5$ $\mathrm{mmol}), t-\mathrm{BuNO}_{2}(2.1 \mathrm{~mL}, 15.6 \mathrm{mmol})$, and DME (65 mL); bright yellow solid $(3.82 \mathrm{~g}, 99 \%)$.

Heck Cross-Coupling Reaction; 1-Bromo-4-[(1E)$3,3,4,4,5,5,6,6,7,7,8,8,9,9,10,10$-heptadecafluorodec-1enyl]naphthalene (5); Typical Procedure

To a stirred suspension of the diazonium salt $3(0.32 \mathrm{~g}, \mathrm{I} \mathrm{mmol})$ and $\mathrm{Pd}(\mathrm{OAc})_{2}$ (11.2 mg, $\left.50 \mathrm{ymol}\right)$ in $\mathrm{MeOH}(3 \mathrm{~mL})$, was added dropwise $\quad 3,3,4,4,5,5,6,6,7,7,8,8,9,9,10,10$-heptadecafluorodec-I-ene $(0.28 \mathrm{~mL}$, I mmol) during $10-15 \mathrm{~min}$. The mixture was then warmed to $40^{\circ} \mathrm{C}$ and allowed to react for additional $90 \mathrm{~min}$. After removal of the solvent, the resulting grey product was passed through a silica gel chromatographiccolumn using a $4: 1$ mixture of
$\mathrm{CH}_{2} \mathrm{Cl}_{2}$-pentane as eluent to afford $5(0.52 \mathrm{~g}, 79 \%)$ as a white solid; $R_{f}=0.73$ (hexane).

${ }^{1} \mathrm{H}$ NMR (360 MHz, CDCI,): $6=8.34-8.31$ (br d, $1 \mathrm{H}_{2 \mathrm{rom}}$ ), 8.048.02 (br d, $1 \mathrm{H}_{\text {arom }}$ ), 7.9-7.95 (br d, $1 \mathrm{H}, \mathrm{CH}=\mathrm{CHC}_{8} \mathrm{~F}_{17}$ ), 7.80-7.82 $\left(\mathrm{d}, 1 \mathrm{H}_{\text {arom }}\right), 7.62-7.68\left(\mathrm{~m}, 2 \mathrm{H}_{\text {arom }}\right), 7.47-7.49\left(\mathrm{~d}, 1 \mathrm{H}_{\text {arom }}\right), 6.22-$ $6.33\left(\mathrm{q}, 1 \mathrm{H}, \mathrm{CH}=\mathrm{CH} \mathrm{C}_{8} \mathrm{~F}_{17}\right)$.

$1-[(1 E)-3,3,4,4,5,5,6,6,7,7,8,8,9,9,10,10$-Heptadecafluorodec-1enyl]-4-nitronaphthalene (6)

Prepared following the diazotization reaction described above: $\mathbf{4}$ $(1.7 \mathrm{~g}, \quad 5.9 \mathrm{mmol}), \quad \mathrm{Pd}(\mathrm{OAc})_{2} \quad(66.5 \mathrm{mg}, \quad 0.3 \mathrm{mmol})$, $3,3,4,4,5,5,6,6,7,7,8,8,9,9,10,10$-heptadecafluorodec-1-ene $\quad(1.56$ $\mathrm{mL}, 5.9 \mathrm{mmol})$, and $\mathrm{MeOH}(20 \mathrm{~mL})$; time: $3 \mathrm{~h}$; yellow solid (3.27 $\mathrm{g}, 90 \%) ; R_{f}=0.61\left(4: 1\right.$ pentane- $\left.\mathrm{CH}_{2} \mathrm{Cl}_{2}\right)$.

${ }^{1} \mathrm{H}$ NMR (360 MHz, CDCI): $6=8.58-8.55(\mathrm{~d}, \mathrm{~J}=8.17 \mathrm{~Hz}, 1$ $\left.\mathrm{H}_{\text {arom }}\right), 8.18-8.2\left(\mathrm{~d}, J=8.17 \mathrm{~Hz}, 1 \mathrm{H}_{\text {arom }}\right), 8.1-8.13(\mathrm{~d}, J=8.17 \mathrm{~Hz}$, $1 \mathrm{H}_{\text {arom }}$ ), 7.95-7.99 (br d, $\left.1 \mathrm{H}, \mathrm{CH}=\mathrm{CHC}_{8} \mathrm{~F}_{17}\right), 7.72-7.81$ (m, 2 $\left.\mathrm{H}_{\text {arom }}\right), 7.67-7.69\left(\mathrm{~d}, 1 \mathrm{H}_{\text {arom }}\right) 6.3-6.41(\mathrm{q}, J=8.17 \mathrm{~Hz}, 1 \mathrm{H}$, $\left.\mathrm{CH}=\mathrm{CHC}_{8} \mathrm{~F}_{17}\right)$.

1-Bromo-4-(3,3,4,4,5,5,6,6,7,7,8,8,9,9,10,10-heptadecafluorodecyl)naphthalene (7)

A solution of $5(4.43 \mathrm{~g}, 6.7 \mathrm{mmol})$ and $\mathrm{Rh} / \mathrm{C}(0.28 \mathrm{~g}, 0.13 \mathrm{mmol})$ in degassed $\mathrm{CH}_{2} \mathrm{Cl}_{2}(35 \mathrm{~mL})$ was placed under 50 bar $\mathrm{H}_{2}$ and the mixture was stirred at r.t. for $24 \mathrm{~h}$. The solvent was evaporated and the residual black solid was then purified by chromatographicfiltration over a silica gel column using $\mathrm{CH}_{2} \mathrm{Cl}_{2}$ as eluent to afford 7 (4 g, $91 \%) ; R_{f}=0.62$ (pentane).

${ }^{1} \mathrm{H}$ NMR (360 MHz, CDCI)): $6=8.31-8.34$ (m, I H $\mathrm{H}_{\text {arom }}$ ), 7.94-7.98 $\left(\mathrm{m}, 1 \mathrm{H}_{\text {arom }}\right), 7.72-7.74\left(\mathrm{~d}, J=7.27 \mathrm{~Hz}, 1 \mathrm{H}_{\text {arom }}\right), 7.61-7.66(\mathrm{~m}, 2$ $\left.\mathrm{H}_{\text {arom }}\right) 7.22-7.24\left(\mathrm{~d}, J=7.70 \mathrm{~Hz}, 1 \mathrm{H}_{\text {arom }}\right), 3.34-3.38(\mathrm{~m}, 2 \mathrm{H}$, $\left.\mathrm{CH}_{2} \mathrm{CH}_{2} \mathrm{C}_{8} \mathrm{~F}_{17}\right), 2.4-2.55$ (m, $2 \mathrm{H}, \mathrm{CH}_{2} \mathrm{CH}_{2} \mathrm{C}_{8} \mathrm{~F}_{17}$ ).

${ }^{13} \mathrm{C} \mathrm{NMR}$ (90.55 MHz, CDCI)): $6=135.2$ (arom), 132.62 (arom), 132.35 (arom), 129.71 (arom), 128.4 (arom), 127.38 (arom), 127.3 (arom), 126.75 (arom), 123.33 (arom), 122.26 (arom), 31.87-32.36 (m, $\left.\mathrm{CH}_{2} \mathrm{CH}_{2} \mathrm{C}_{8} \mathrm{~F}_{17}\right), 23.5-23.6\left(\mathrm{~m}, \mathrm{CH}_{2} \mathrm{CH}_{2} \mathrm{C}_{8} \mathrm{~F}_{17}\right)$.

\section{4-(3,3,4,4,5,5,6,6,7,7,8,8,9,9,10,10-Heptadecafluorodecyl)-1- naphthylamine $(8)$}

A solution of $6(0.93 \mathrm{~g}, 1.5 \mathrm{mmol})$ and $\mathrm{Pd} / \mathrm{C}(0.08 \mathrm{~g}, 75 \mathrm{ymol}, 10 \%$ $\mathrm{Pd}$ ) in degassed THF-MeOH (I:I, $15 \mathrm{~mL}$ ) was placed under 3 bar of $\mathrm{H}_{2}$ and the mixture was stirred at r.t. for $24 \mathrm{~h}$. The solvent was evaporated and the residual black solid was then purified by chromatographic filtration over a silica gel column with pentane$\mathrm{CH}_{2} \mathrm{Cl}_{2}(2: 1)$ as eluent $(0.83 \mathrm{~g}, 94 \%) ; R_{f}=0.21 \quad(2: 1$ pentane$\mathrm{CH}_{2} \mathrm{Cl}_{2}$ ),

${ }^{1} \mathrm{H}$ NMR $\left(360 \mathrm{MHz}, \mathrm{C}_{6} \mathrm{D}_{6}\right): 6=7.74-7.76\left(\mathrm{~d}, J=8.17 \mathrm{~Hz}, 1 \mathrm{H}_{\text {arom }}\right)$, $7.49-7.51\left(\mathrm{~d}, J=8.17 \mathrm{~Hz}, I \mathrm{H}_{\text {arom }}\right), 7.2-7.35\left(\mathrm{~m}, 2 \mathrm{H}_{\text {arom }}\right), 6.81-6.83$ $\left(\mathrm{d}, J=7.74 \mathrm{~Hz}, 1 \mathrm{H}_{\text {arom }}\right), 6.29-6.31\left(\mathrm{~d}, J=7.74 \mathrm{~Hz}, 1 \mathrm{H}_{\text {arom }}\right), 3.3(\mathrm{br}$ s, $2 \mathrm{H}, \mathrm{NH}), 3.06-3.1\left(\mathrm{~m}, 2 \mathrm{H}, \mathrm{CH}_{2} \mathrm{CH}_{2} \mathrm{C}_{8} \mathrm{~F}_{17}\right), 2.12-2.24(\mathrm{~m}, 2 \mathrm{H}$, $\mathrm{CH}_{2} \mathrm{CH}_{2} \mathrm{C}_{8} \mathrm{~F}_{17}$ ).

\section{Palladium-Catalyzed Amination Reaction; $N, N$-Bis [4-}

$(3,3,4,4,5,5,6,6,7,7,8,8,9,9,10,10$-heptadecafluorodecyl)-1-naphthyl]amine (10); Typical Procedure

A Schlenk flask was charged with $7(5.54 \mathrm{~g}, 8.48 \mathrm{mmol}), 8(5 \mathrm{~g}$, $8.48 \mathrm{mmol}), \mathrm{Pd}(\mathrm{OAc})_{2}(190 \mathrm{mg}, 0.82 \mathrm{mmol}), t-\mathrm{Bu}_{3} \mathrm{P}(0.7 \mathrm{~g}, 3.5$ $\mathrm{mmol}), t$-BuONa $(1.23 \mathrm{~g}, 12.8 \mathrm{mmol})$, and toluene $(150 \mathrm{~mL})$. The reaction was refluxed for $2 \mathrm{~d}$ under argon. The dark brown solution was diluted with aq sat. $\mathrm{NH}_{4} \mathrm{Cl}(30 \mathrm{~mL})$ and extracted with $\mathrm{Et}_{2} \mathrm{O}$ $(3 \times 25 \mathrm{~mL})$. The combined organic layers were washed with $\mathrm{H}_{2} \mathrm{O}$ $(3 \times 25 \mathrm{~mL})$, dried $\left(\mathrm{K}_{2} \mathrm{CO}_{3}\right)$, and filtered. Removal of the solvent yielded a brown product which was chromatographed using a mixture of pentane- $\mathrm{CH}_{2} \mathrm{Cl}_{2}(9: 1)$ to afford $9(7 \mathrm{~g}, 71 \%)$ as a faint yellow product; $R_{f}=0.41\left(9: 1\right.$ pentane $\left.-\mathrm{CH}_{2} \mathrm{Cl}_{2}\right)$. 
${ }^{1} \mathrm{H}$ NMR $\left(360 \mathrm{MHz}, \mathrm{C}_{6} \mathrm{D}_{6}\right): 6=7.94-7.96\left(\mathrm{~d}, J=9.05 \mathrm{~Hz}, 2 \mathrm{H}_{\text {arom }}\right)$, $7.81-7.83\left(\mathrm{~d}, J=8.35 \mathrm{~Hz}, 2 \mathrm{H}_{\text {arom }}\right), 7.23-7.34\left(\mathrm{~m}, 4 \mathrm{H}_{\text {arom }}\right), 6.80$ $6.82\left(\mathrm{~d}, J=7.70 \mathrm{~Hz}, 2 \mathrm{H}_{\text {arom }}\right), 6.75-6.77\left(\mathrm{~d}, \mathbf{J}=7.70 \mathrm{~Hz}, 2 \mathrm{H}_{\text {arom }}\right)$, 5.8 (br s, $1 \mathrm{H}, \mathrm{NH}$ ), 3.09-3.13 (m, $\left.4 \mathrm{H}, \mathrm{CH}_{2} \mathrm{CH}_{2} \mathrm{C}_{8} \mathrm{~F}_{17}\right), 2.19-2.24$ (m, $4 \mathrm{H}, \mathrm{CH}_{2} \mathrm{CH}_{2} \mathrm{C}_{8} \mathrm{~F}_{17}$ ).

${ }^{13} \mathrm{C}$ NMR (90.55 MHz, $\left.\mathrm{C}_{6} \mathrm{D}_{6}\right): 6=140.3$ (arom), 132.73 (arom), 131.91 (arom), 128.15 (arom), 126.82 (arom), 126.8 (arom), 125.66 (arom), 123.71 (arom), 123.31 (arom), 115.73 (arom), 31.4 (m, $\left.\mathrm{CH}_{2} \mathrm{CH}_{2} \mathrm{C}_{8} \mathrm{~F}_{17}\right), 23.34\left(\mathrm{~m}, \mathrm{CH}_{2} \mathrm{CH}_{2} \mathrm{C}_{8} \mathrm{~F}_{17}\right)$.

ESI-MS $\left(\mathrm{HCO}_{2} \mathrm{H}-\mathrm{THF}\right): m / z(\%)=1206\left(\left[\mathrm{M}+\mathrm{CO}_{2}\right]+35\right), 1162$ $\left([\mathrm{M}+\mathrm{H}]^{+}, 100\right), 288(22)$.

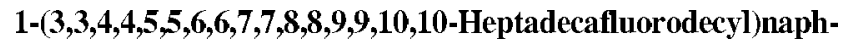
thalene (11)

$R_{f}=0.66$ (9:1 hexane).

${ }^{1} \mathrm{H}$ NMR $\left(500 \mathrm{MHz}, \mathrm{C}_{6} \mathrm{D}_{6}\right): 6=7.72-7.74\left(\mathrm{~m}, \mathrm{I} \mathrm{H} \mathrm{H}_{\text {arom }}\right), 7.64-7.66$ ( $\left.\mathrm{m}, 1 \mathrm{H}_{\mathrm{arom}}\right), 7.54-7.56\left(\mathrm{brd}, 1 \mathrm{H}_{\mathrm{arom}}\right), 7.24-7.28\left(\mathrm{~m}, 2 \mathrm{H}_{\mathrm{arom}}\right), 7.14$ 7.17 (br d, $1 \mathrm{H}_{\text {arom }}$ ), 6.87-6.89 (br d, $\left.1 \mathrm{H}_{\text {arom }}\right), 3.06-3.1(\mathrm{~m}, 2 \mathrm{H}$, $\left.\mathrm{CH}_{2} \mathrm{CH}_{2} \mathrm{C}_{8} \mathrm{~F}_{17}\right), 2.09-2.2\left(\mathrm{~m}, 2 \mathrm{H}, \mathrm{CH}_{2} \mathrm{CH}_{2} \mathrm{C}_{8} \mathrm{~F}_{17}\right)$.

${ }^{13} \mathrm{C} \mathrm{NMR}\left(125.77 \mathrm{MHz}, \mathrm{C}_{6} \mathrm{D}_{6}\right.$ ): $6=135.09$ (arom), 134.46 (arom), 131.85 (arom), 129.33 (arom), 128.29 (arom), 126.64 (arom), 126.44 (arom), 125.98 (arom), 125.75 (arom), 123.04 (arom), 111.06-120.80(m, CF $), 31.9-31.3\left(\mathrm{~m}, \mathrm{CH}_{2} \mathrm{CH}_{2} \mathrm{C}_{8} \mathrm{~F}_{17}\right), 23.58$.

$N$-[4',8-Bis $(3,3,4,4,5,5,6,6,7,7,8,8,9,9,10,10$-heptadecafluorodecyl)-1,1'-binaphthalen-4-yl]- $N$-[4$(3,3,4,4,5,5,6,6,7,7,8,8,9,9,10,10$-heptadecafluorodecyl)-1-naphthyl]amine (12)

The reaction was carried out following the Pd-catalyzed amination reaction: 10 (116 mg, $0.1 \mathrm{mmol}), 7(66 \mathrm{mg}, 0.1 \mathrm{mmol}), \mathrm{Pd}(\mathrm{OAc})_{2}$ (1.12 mg, $5 \mu \mathrm{mol}), t-\mathrm{Bu} 3 \mathrm{P}(4 \mathrm{mg}, 20 \mu \mathrm{mol}), \mathrm{Cs}_{2} \mathrm{CO}_{3}(100 \mathrm{mg}, 0.3$ $\mathrm{mmol})$, and degassed xylene $(10 \mathrm{~mL})$; yield: $27 \mathrm{mg}(16 \%)$.

${ }^{1} \mathrm{H}$ NMR $\left(500 \mathrm{MHz}, \mathrm{C}_{6} \mathrm{D}_{6}\right): 6=8.23-8.25\left(\mathrm{~m}, 1 \mathrm{H}_{\text {arom }}\right), 7.92-7.94$ (d, $\left.1 \mathrm{H}_{\mathrm{arom}}\right), 7.88-7.9\left(\mathrm{~m}, 1 \mathrm{H}_{\mathrm{arom}}\right), 7.72\left(\mathrm{br} \mathrm{s}, \mathrm{I} \mathrm{H}_{\text {arom }}\right), 7.62-7.63(\mathrm{~d}$, $1 \mathrm{H}_{\text {arom }}$ ), 7.52 (br s, $1 \mathrm{H}_{\text {arom }}$ ), 7.31-7.36 (overlapped peaks, $3 \mathrm{H}_{\text {arom }}$ ), 7.2-7.25 (m, $\left.4 \mathrm{H}_{\text {arom }}\right), 6.98-7.01$ (br t, $\left.1 \mathrm{H}_{\text {arom }}\right), 6.63$ (br s, $2 \mathrm{H}_{\text {arom }}$ ), 6.48-6.49 (d, I H $\left.\mathrm{H}_{\text {arom }}\right), 5.96(\mathrm{~s}, \mathrm{I} \mathrm{H}, \mathrm{NH}), 3.19-3.3(\mathrm{~m}, 2 \mathrm{H}$, $\left.\mathrm{CH}_{2} \mathrm{CH}_{2} \mathrm{C}_{8} \mathrm{~F}_{17}\right), 2.89-3.02\left(\mathrm{~m}, 4 \mathrm{H}, \mathrm{CH}_{2} \mathrm{CH}_{2} \mathrm{C}_{8} \mathrm{~F}_{17}\right), 2.31-2.39(\mathrm{~m}, 2$ $\left.\mathrm{H}, \mathrm{CH}_{2} \mathrm{CH}_{2} \mathrm{C}_{8} \mathrm{~F}_{17}\right), 1.94-2.07$ (m, $\left.4 \mathrm{H}, \mathrm{CH}_{2} \mathrm{CH}_{2} \mathrm{C}_{8} \mathrm{~F}_{17}\right)$.

EI-MS: $m / z(\%)=1733(\mathrm{M}+70), 1714\left([\mathrm{M}-\mathrm{F}]^{++}, 8\right), 1300([\mathrm{M}-$ $\left.\left.\mathrm{CH}_{2}\left(\mathrm{R}_{\mathrm{f}}\right)_{8}\right]^{++}, 100\right), 156(60)$.

$N, N$-Bis[4- $(3,3,4,4,5,5,6,6,7,7,8,8,9,9,10,10$-heptadecafluorodecyl)-1-naphthyl]- $N$ - $[4-(3,3,4,4,5,5,6,6,7,7,8,8,9,9,10,10$-heptadecafluorodecyl)phenyl]amine (13)

Prepared following the amination reaction described as above: 9 (116 mg, $0.1 \mathrm{mmol})$, 1-bromo-4-(3,3,4,4,5,5,6,6,7,7,8,8,9,9,10,10heptadecafluorodecyl)benzene ( $60 \mathrm{mg}, 0.1 \mathrm{mmol}), \mathrm{Pd}(\mathrm{OAc})_{2}(1.12$ $\mathrm{mg}, 5 \mu \mathrm{mol}), t$-Bu 3 P (4 mg, $20 \mu \mathrm{mol}), t$-BuONa (15 mg, 0.15 $\mathrm{mmol})$, and toluene $(5 \mathrm{~mL})$; time: $3.5 \mathrm{~d}$; glassy colorless solid; yield: $108 \mathrm{mg}(64 \%) ; \mathrm{mp}>450^{\circ} \mathrm{C} ; R_{f}=0.65\left(9: 1\right.$ pentane $-\mathrm{CH}_{2} \mathrm{Cl}_{2}$ ). ${ }^{1} \mathrm{H}$ NMR (360 MHz, THF- $\left.d_{8}\right): 6=8.268 .28(\mathrm{~d}, J=8.17 \mathrm{~Hz}, 2$ $\left.\mathrm{H}_{\text {arom }}\right), 8.17-8.19\left(\mathrm{~d}, \mathbf{J}=8.64 \mathrm{~Hz}, 2 \mathrm{H}_{\text {arom }}\right), 7.63-7.68(\mathrm{t}, \mathbf{J}=7.7 \mathrm{~Hz}$, $\left.2 \mathrm{H}_{\text {arom }}\right), 7.45-7.48\left(\mathrm{~m}, 4 \mathrm{H}_{\text {arom }}\right.$ and $\left.\mathrm{C}_{6} \mathrm{H}_{4}\right), 7.25-7.27(\mathrm{~d}, J=7.7 \mathrm{~Hz}$, $\left.2 \mathrm{H}, \mathrm{C}_{6} \mathrm{H}_{5}\right), 7.13-7.15\left(\mathrm{~d}, J=8.64 \mathrm{~Hz}, 2 \mathrm{H}_{\mathrm{arom}}\right), 6.73-6.76(\mathrm{~d}$, $\mathbf{J}=8.17 \mathrm{~Hz}, 2 \mathrm{H}_{\text {arom }}$ ), 3.50-3.54 (br t, $4 \mathrm{H}, \mathrm{CH}_{2} \mathrm{CH}_{2} \mathrm{C}_{8} \mathrm{~F}_{17}$ ), 2.92 2.96 (br t, $2 \mathrm{H}, \mathrm{CH}_{2} \mathrm{CH}_{2} \mathrm{C}_{8} \mathrm{~F}_{17}$ ), 2.67-2.78 (m, $4 \mathrm{H}, \mathrm{CH}_{2} \mathrm{CH}_{2} \mathrm{C}_{8} \mathrm{~F}_{17}$ ), 2.53-2.62 (m, $\left.2 \mathrm{H}, \mathrm{CH}_{2} \mathrm{CH}_{2} \mathrm{C}_{8} \mathrm{~F}_{17}\right)$.

${ }^{1} \mathrm{H}$ NMR $\left(360 \mathrm{MHz}, \mathrm{C}_{6} \mathrm{D}_{6}\right): 6=8.45-8.48\left(\mathrm{~d}, \mathrm{~J}=8.64 \mathrm{~Hz}, 2 \mathrm{H}_{\text {arom }}\right)$, $7.73-7.76\left(\mathrm{~d}, J=8.17 \mathrm{~Hz}, 2 \mathrm{H}_{\text {arom }}\right), 7.21-7.23\left(\mathrm{~d}, 2 \mathrm{H}_{\text {arom }}\right), 7.09$ $7.17\left(\mathrm{~m}, 4 \mathrm{H}_{\text {aron }}\right.$ and $\left.\mathrm{C}_{6} \mathrm{H}_{5}\right), 6.8-6.84\left(\mathrm{t}, 4 \mathrm{H}_{\text {arom }}\right), 6.62-6.64(\mathrm{~d}$, $J=8.17 \mathrm{~Hz}, 2 \mathrm{H}_{\text {arom }}$ ), 3.05-3.09 (br t, $4 \mathrm{H}, \mathrm{CH}_{2} \mathrm{CH}_{2} \mathrm{C}_{8} \mathrm{~F}_{17}$ ), 2.492.53 (br t, $\left.2 \mathrm{H}, \mathrm{CH}_{2} \mathrm{CH}_{2} \mathrm{C}_{8} \mathrm{~F}_{17}\right), 2.08-2.23\left(\mathrm{~m}, 4 \mathrm{H}, \mathrm{CH}_{2} \mathrm{CH}_{2} \mathrm{C}_{8} \mathrm{~F}_{17}\right.$ ), 1.83-1.98 (m, $\left.2 \mathrm{H}, \mathrm{CH}_{2} \mathrm{CH}_{2} \mathrm{C}_{8} \mathrm{~F}_{17}\right)$.
${ }^{13} \mathrm{C}$ NMR (90.55 MHz, $\left.\mathrm{C}_{6} \mathrm{D}_{6}\right): 6=133.92$ (arom), 133.35 (arom), 131.45 (arom), 129.8 (arom), 127.28 (arom), 127.3 (arom), 127.13 (arom), 126.08 (arom), 125.27 (arom), 124.38 (arom), 33.3 (br s, $\mathrm{CH}_{2} \mathrm{CH}_{2} \mathrm{C}_{8} \mathrm{~F}_{17}$ ), 32.51 (br s, $\left.\mathrm{CH}_{2} \mathrm{CH}_{2} \mathrm{C}_{8} \mathrm{~F}_{17}\right), 23.95$ (br s, $\left.\mathrm{CH}_{2} \mathrm{CH}_{2} \mathrm{C}_{8} \mathrm{~F}_{17}\right)$.

ESI-MS $\left(\mathrm{HCO}_{2} \mathrm{H}-\mathrm{THF}\right): m / z(\%)=1732\left(\left[\mathrm{M}+\mathrm{HCO}_{2}\right]^{+}, 55\right), 1683$ $\left(\mathrm{M}^{+}, 100\right)$

EI-MS: $m / z(\%)=1683\left(\mathrm{M}^{+}, 100\right), 1664\left([\mathrm{M}-\mathrm{F}]^{+}, 40\right), 1250$ $\left(\left[\mathrm{M}-\mathrm{CH}_{2} \mathrm{C}_{8} \mathrm{~F}_{17}\right]^{++}, 45\right), 789(10)$.

$N, N, N^{\prime}, N^{\prime}$-Tetrakis[4-(3,3,4,4,5,5,6,6,7,7,8,8,9,9,10,10-heptadecafluorodecyl)-1-naphthyl]-1,1'-biphenyl-4,4'-diamine (14)

Prepared following the amination reaction described above; 9 (3.5 $\mathrm{g}, 3 \mathrm{mmol}), 4,4^{\prime}$-dibromobiphenyl $(0.46 \mathrm{~g}, 1.47 \mathrm{mmol}), \mathrm{Pd}(\mathrm{OAc})_{2}$ $(64 \mathrm{mg}, 0.29 \mathrm{mmol}), t-\mathrm{Bu}_{3} \mathrm{P}(71 \mathrm{mg}, 0.35 \mathrm{mmol}), t-\mathrm{BuONa}(0.38 \mathrm{~g}$, $3.95 \mathrm{mmol})$, and toluene $(100 \mathrm{~mL})$; time: $7 \mathrm{~d}$. After washing the mixture with aq sat. $\mathrm{NH}_{4} \mathrm{Cl}(25 \mathrm{~mL})$ and aq $5 \%$ solution of $\mathrm{KCN}(10$ $\mathrm{mL}$ ), the organic phase was filtered and the faint brown precipitate was suspended in $\mathrm{CH}_{2} \mathrm{Cl}_{2}$, sonicated for 15-20 min and collected by suction filtration. The precipitate was purified by successive washings with $\mathrm{CH}_{2} \mathrm{Cl}_{2}$ and $\mathrm{Et}_{2} \mathrm{O}$ affording 11 (2.07 g, 57\%) as an offwhite solid; $\mathrm{mp} 242^{\circ} \mathrm{C}$.

${ }^{1} \mathrm{H}$ NMR $\left(360 \mathrm{MHz}, \mathrm{THF}-d_{8}\right): 6=8.16-8.18(\mathrm{~d}, J=8.64 \mathrm{~Hz}, 4$ $\left.\mathrm{H}_{\mathrm{arom}}\right), 8.05-8.07\left(\mathrm{~d}, J=8.6 \mathrm{~Hz}, 4 \mathrm{H}_{\mathrm{arom}}\right), 7.51-7.55(\mathrm{t}, J=7.27 \mathrm{~Hz}$, $\left.4 \mathrm{H}_{\text {arom }}\right), 7.34-7.37\left(\mathrm{~m}, 8 \mathrm{H}_{\text {arom }}\right), 7.29-7.31(\mathrm{~d}, J=8.17 \mathrm{~Hz}, 4 \mathrm{H}$, $\left.\mathrm{C}_{6} \mathrm{H}_{4}\right), 6.65-6.67\left(\mathrm{~d}, J=8.17 \mathrm{~Hz}, 4 \mathrm{H}, \mathrm{C}_{6} \mathrm{H}_{4}\right), 2.562 .68(\mathrm{~m}, 8 \mathrm{H}$, $\left.\mathrm{CH}_{2} \mathrm{CH}_{2} \mathrm{C}_{8} \mathrm{~F}_{17}\right)$.

ESI-MS $\left(\mathrm{HCO}_{2} \mathrm{H}-\mathrm{THF}\right): m / z(\%)=2473\left(\mathrm{M}^{+}, 100\right), 1957(58), 686$ (48).

EI-MS: $m / z=2473(\mathrm{M}+100 \%)$.

\section{Acknowledgment}

We thank the Swiss National Science Foundation National Research Program: Supramolecular Functional Materials (NRP 47057425) for supporting this work.

\section{References}

(1) Tang, C. W.; Van Slyke, S. A. Appl. Phys. Lett. 1987, 51, 913.

(2) Kuwabara, Y.; Ogawa, H.; Inada, H.; Noma, N.; Shirota, Y. Adv. Mater. 1994, 6, 677.

(3) Zilker, S. J. ChemPhysChem 2000, I, 72.

(4) Bach, U.; Lupo, D.; Comte, P.; Moser, J. E.; Weissortel,F.; Salbeck, J.; Spreitzer, H.; Grätzel, M. Nature 1998,395, 583.

(5) Law, K. Y. Chem. Rev. 1993, 93, 449.

(6) Thelakkat, M. Macromol. Mater. Eng. 2002,287,442; and references cited therein.

(7) Naito, K.; Miura, A. J. Phys. Chem. 1993, 97, 6240.

(8) Horgan, A. M. Xerox Corporation; US Patent 4047948, 1977; Chem. Abstr. 1977, 88, 43748.

(9) Goodbrand, H. B.; Hu, N.-X. J. Org. Chem. 1999, 64, 670.

(10) Shirota, Y.; Kobata, T.; Noma, N. Chem. Lett. 1989, 7, 1145 ; and references cited therein.

(I I) Plater, M. J.; McKay, M.; Jackson, T. J. Chem. Soc., Perkin Trans. $12000,2695$.

(12) Tanaka, H.; Tokito, S.; Taga, Y.; Okada, A. Chem. Commun. 1996, 2175.

(13) Louie, J.; Hartwig, J. F. J. Am. Chem. Soc. 1997,119, 11695.

(14) Thelakkat, M.; Hagen, J.; Haarer, D.; Schmidt, H.-W.Synth. Met. 1999,102, 1125.

(15) Visjager, J.; Tervort, T. A.; Smith, P. Polymer 1999, 40, 4533. 
(16) Darses, S.; Pucheault, M.; Genêt, J.-P. Eur. J. Org. Chem. 2001, 66, 1121.

(17) Doyle, M. P.; Bryker, W. J. J. Org. Chem. 1979, 44, 1572.

(18) Hartwig, J. F. Angew. Chem. Int. Ed. 1998, 37, 2046.

(19) Enokida, T.; Tamano, M.; Okutsu, S. Eur. Pat. Appl. EP 765106A2, 1997; Chem. Abstr. 1997, 126, 310317.
(20) Tamano, M.; Okutsu, S. US Patent US 005968675,1999; Chem. Abstr. 1999, 131,294207.

(21) Hartwig, J. F.; Goodson, F. E.; Louie, J.; Hauck, S. Polym. Mat. Sci. Eng. 1999, 80, 41.

(22) Hartwig, J. F. Polym. Prep. 2000, 41, 420.

(23) Pangborn, A. B.; Giardello, M. A.; Grubbs, R. H.; Rosen, R. K.; Timmers, F. J. Organometallics 1996, 15, 1518. 